\title{
AN ESTIMATION-BASED APPROACH TO MULTIPLE-INPUT MULTIPLE-OUTPUT (MIMO) CHANNEL EQUALIZATION
}

\author{
Ardavan Maleki Tehrani, Babak Hassibi*, John Cioff \\ STAR Lab \\ 350 Serra Mall, Packard Bldg. 360 \\ Stanford, CA, USA \\ ${ }^{*}$ Mathematics of Communications Dept., Bell Labs, Lucent Technologies. \\ ardavan@leland. stanford.edu,
}

\begin{abstract}
The purpose of this paper is to propose and investigate a new approach to implementing a spatio-temporal Decision Feedback Equalizer (DFE) for MIMO (MultipleInput Multiple-Output) channels. A system with an array of $n$ transmit and $m$ receiver antennas where $(m \geq$ $n$ ) is assumed. Both finite-length (Finite Horizon) and infinite-length (Infinite Horizon) MIMO Decision Feedback Equalizers are considered. We also assume an ISI (Inter-Symbol-Interference) MIMO channel, which mean$s$ the channel matrix elements are frequency selective.

For the infinite-length case the DFE problem leads to solving a matrix spectral factorization. For the finitelength case the DFE problem leads to solving a corresponding Cholesky factorization.

Using the estimation-based spectral factorization we have shown that the solution to the infinite-length MIMO DFE is not unique. In the finite-length case the estimationbased approach leads to a recursive algorithm to perform the Cholesky factorization. The proposed recursive algorithm has low complexity and is also simple to implement. Moreover it leads to a closed form solution for the MIMO DFE matrices.
\end{abstract}

\section{INTRODUCTION}

Although the problem of channel equalization has been extensively studied in the literature the growth of wireless communications has presented new challenges. In particular, the introduction of space-time coding ([1],[2],[3],[4]) and application of antenna arrays at both transmitter and receiver ([5],[6]) has encouraged new research on equalization techniques for so called Multiple-Input Multiple-Output channels.

The authors would like to thank NSF and SK Telecom for their support and funding.
It must be noted that the problem of MIMO equalization has been readily studied in the literature ([7],[8],[9],[10],[11]). The purpose of this paper is to propose and investigate a new estimation-based approach to spatio-temporal equalization for MIMO (Multiple-Input Multiple-Output) channel equalization. We will consider a Decision Feedback Equalizers (DFE) structure. We have considered a system with $n$ transmit and $m(m \geq n)$ receiver antennas, therefore an $m \times n$ channel matrix. Each element of the resulting MIMO channel is considered to be frequency selective. The channel is also assumed to be AWGN (Additive White Gaussian Noise). The output of the $m$ receiver antennas, after passing through the matched filter, are fed into the matrix equalizer(s). The DFE equalizer consists of two matrix of linear filters each with maximum $L$ taps, one for the feedforward section and one for the feedback section. A perfect channel knowledge at the receiver is assumed.

For the infinite-length case the DFE problem leads to solving a matrix spectral factorization. For the finite-length case the DFE problem leads to solving a corresponding $\mathrm{C}$ holesky factorization. We present estimation-based solutions to the vector spectral factorization and the corresponding Cholesky factorization, and these solutions are used to solve the corresponding formulated DFE problems.

We will show closed form solutions for the MIMO DFE matrices, which are due to the state-space structure used in solving the problem. None of the readily studied MIMO equalization methods lead to closed form solutions.

The paper is formatted as following. In section 2 we will discuss the MIMO DFE problem in two parts, the infinte horizon (inifnite length) case and the finite horizon (finite length) case. In section 2.1 we will discuss the solution to the infinite length MIMO DFE. We will first present the matrix spectral factorization solution using a state space model in subsection 2.1.1. Then we will present the solution to the infinite length MIMO DFE problem in subsection 2.1.2. Correspondingly in section 2.2 we will discuss the solution 
to the finite length MIMO DFE. The matrix Cholesky factorization solution using a state space model is presented in subsection 2.2.1 and solution to the finite length MIMO DFE is presented in subsection 2.2.2. We finally conclude in Section 3.

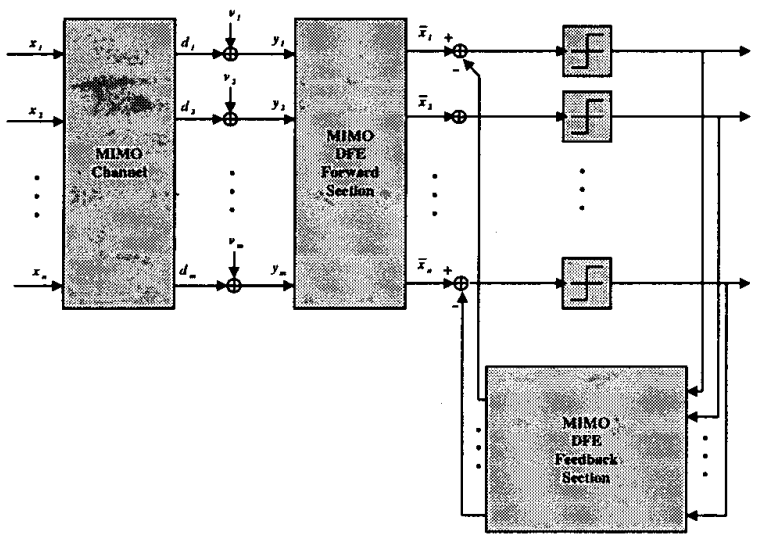

Figure 1: Block diagram of the MIMO DFE

\section{MIMO DECISION FEEDBACK EQUALIZATION (MIMO DFE)}

The structure of the MIMO DFE is shown in figure (1). Note that the variables in the figure are just used to depict input$s$ and outputs, and do not correspond to any specific variable in the paper. As in the scalar case the MIMO DFE equalizer consists of a feedforward, a feedback and a decision (slicer) section. Knowing the channel matrix the DFE problem is how to find the feedback and the feedforward matrices. In this paper the derivations will be based on the Minimum Mean Squared Error (MMSE) formulation. We shall note that MMSE formulation is stochastic versus the Least Square formulation which is deterministic. We will be considering two cases:

- The Infinite Horizon Case

- The Finite Horizon Case

\subsection{Infinite Horizon}

In the Infinite Horizon case the length of the DFE filters can be arbitrarily large, corresponding to the scalar infinite lenght DFE. The following derivations are discussed in [12], and we will just mention the final results here. Lets assume $\mathbf{Q}(z)$ represents the channel matrix convolved with the matched filter matrix, and lets define $\tilde{\mathbf{Q}}(z)$ :

$$
\tilde{\mathbf{Q}}(z)=\mathbf{Q}(z)+\frac{N_{0} / 2}{\epsilon_{x}} I
$$

where $N_{0} / 2$ is the noise spectral density per dimension. Lets assume $\tilde{\mathbf{Q}}(z)$ has spectral factorization

$$
\tilde{\mathbf{Q}}(z)=\gamma G(z) G^{*}\left(z^{-*}\right)
$$

Then the MMSE feedback matrix $\mathbf{B}(z)$ is found as a result of the above spectral factorization to be

$$
\mathbf{B}(z)=G(z)^{-1}
$$

The feedforward matrix $\mathbf{W}(\mathbf{z})$ is then found from $\mathbf{B}(\mathbf{z})$ (please refer to [12]).

\subsubsection{Matrix Spectral Factorization}

As discussed in the previous section in order to find the DFE matrices $\mathbf{B}(\mathbf{z})$ and $\mathbf{W}(\mathbf{z})$ we need to solve a matrix spectral factorization problem. In this section we will presend a solution to the matrix spectral factorization using a state space approach used in [13]. We will present a matrix spectral factorization solution for a vector process whose statistics is described by a state space model. Then in the next section we will formulate our DFE problem, and will use the development in this section to solve the corresponding DFE spectral factorization problem.

Lets assume a process $\left\{y_{i}\right\}$ with a time-invariant spacetime model

$$
\left\{\begin{array}{c}
x_{i+1}=F x_{i}+G u_{i} \\
y_{i}=H x_{i}+v_{i}
\end{array}\right.
$$

where $F \in C^{n \times n}, G \in C^{n \times m}$, and $H \in C^{p \times n}$ are known time-independent matrices, and $\left\{u_{i}\right\}$ and $\left\{v_{i}\right\}$ are zero-mean jointly stationary vector random variables that, along with the zero-mean random variable $x_{0}$ satisfy the conditions

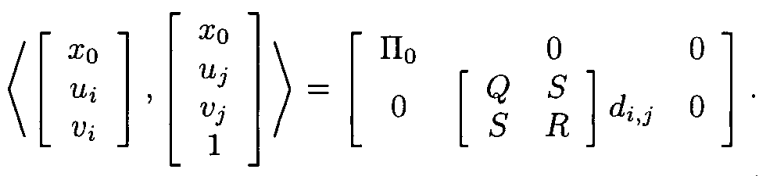

As seen the driving processes $\left\{u_{i}\right\}$ and $\left\{v_{i}\right\}$ are white(temporally uncorrelated). For more information on the above statespace description please refer to [13].

When the process $\left\{y_{i}\right\}$ is stationary it can be shown that it has an exponentially decaying covariance function. We can therefore introduce its $z$-power spectral density matrix, $S_{y}\{z\}$, as the bilateral z-transform of $R_{y}(k)$,

$$
S_{y}\{z\}=\sum_{0}^{\infty} R_{y}(k) z^{-k}
$$


The state-space model (4) allows us to give some explicit formulas for $S_{y}\{z\}$. It can be shown that assuming $\left\{y_{i}\right\}$ being stationary then the z-spectrum is given by,

$$
S_{y}\{z\}=K(z)\left[\begin{array}{cc}
G Q G^{*} & G S \\
S^{*} G^{*} & R
\end{array}\right] K^{*}\left(z^{-*}\right)
$$

where

$$
K\{z\}=\left[H(z I-F)^{-1} \quad I\right]
$$

It can be shown [14] that the z-spectrum $S_{y}\{z\}$ is not unique and there is an equivalence class for the input covariance matrices that give rise to the same z-spectrum, $S_{y}\{z\}$. In fact the non-uniqueness of the input covariance matrices was exploited to obtain the above spectral factorization of $S_{y}\{z\}$.

Given the above derivation we will summarize the matrix spectral factorization results in the following theorem referring from [14].

Theorem 1 (Matrix Spectral Factorization) Consider the time-invariant state-space model 4. Suppose that $F$ is stable and that the initial state covariance matrix is such that

$$
\Pi_{0}=\bar{\Pi}=F \bar{\Pi} F^{*}+G Q G^{*},
$$

so that the output process $\left\{y_{i}, i>0\right\}$ is stationary. Assume further that $\{F, H\}$ is detectable, $\left\{F-G S R^{-1} H, G(Q-\right.$ $\left.\left.S R^{-1} S^{*}\right)^{1 / 2}\right\}$ is stabilizable, $R>0$ and

$$
\left[\begin{array}{ll}
Q & S \\
S^{*} & R
\end{array}\right]>0
$$

Then the canonical spectral factorization of the z-spectral density function $S_{y}\{z\}$,

$$
S_{y}\{z\}=K(z)\left[\begin{array}{cc}
G Q G^{*} & G S \\
S^{*} G^{*} & R
\end{array}\right] K^{*}\left(z^{-*}\right)
$$

is given by

$$
S_{y}\{z\}=L(z) R_{e} L^{*}\left(z^{-*}\right)
$$

where $K(z)$ is given in (8) and the monic, causal and causally invertible transfer matrix $L(z)$ is given by

$$
L(z)=H(z I-F)^{-1} K_{P}+I
$$

with

$$
R_{e}=R+H P H^{*} \text { and } K_{p}=\left(F P H^{*}+G S\right) R_{e}^{-1}
$$

where $P$ is the unique positive semi-definite solution to the $D A R E$

$$
P=F P F^{*}+G Q G^{*}-K_{p} R_{e} K_{p}^{*} .
$$

Moreover, $F-K_{p} H$ is stable, which implies that $L^{-1}(z)$ exists and is stable.

\subsubsection{Infinite Horizon DFE solution}

As discussed in section 2.1 the solution for the feedback section is found from spectral factorization of $\tilde{\mathbf{Q}}(z)$. In the previous section (2.1.1), we showed how to factorize $S_{y}\{z\}$ the z-spectrum of the stationary process $\left\{y_{i}\right\}$ which is described by the state-space model (4). In order to use the solution obtained in the previous section we will equate

$$
S_{y}\{z\} \triangleq \tilde{\mathbf{Q}}(z) .
$$

We have the solution to factorizing $S_{y}\{z\}$. Now we have to find the state-space model that leads to (10).

We will present the results and will leave the proof to [15]. It can be shown [15] that the following choices for the state-space model (4) will lead to (10):

$$
\begin{aligned}
F & =\left[\begin{array}{ccccc}
0 & 0 & \cdots & & 0 \\
I_{m} & 0 & \cdots & & \vdots \\
0 & I_{m} & 0 & & \\
\vdots & & \ddots & \ddots & \vdots \\
0 & \cdots & 0 & I_{m} & 0
\end{array}\right], \\
H & =\left[\begin{array}{llll}
\tilde{\mathbf{Q}}(1) & \tilde{\mathbf{Q}}(2) & \cdots & \tilde{\mathbf{Q}}(l)
\end{array}\right], \\
G & =\left[\begin{array}{lll}
I_{m} & 0 \cdots 0
\end{array}\right]^{\prime} \\
Q & =0, \quad R=\tilde{\mathbf{Q}}(0)+\frac{I}{S N R}, \quad S=I .
\end{aligned}
$$

where $l$ is the length of $\tilde{\mathbf{Q}}(z)$ in time. Therefore the DFE feedback matrix is $\mathbf{B}(z)=L(z)^{-1}$ where $L(z)$ is found from Theorem 1 using the above state-space model parameters. The feedforward matrix $\mathbf{W}(\mathbf{z})$ is then found from $\mathbf{B}(\mathbf{z})$ (please refer to [12]).

\subsection{Finite Horizon}

In the finite Horizon case the length of the DFE filters are finite, corresponding to the scalar finite length DFE. The DFE structure is again as shown in Figure (1). As in the previous section we will leave the derivations to [12], and will just mention the results. As in the infinite length case the DFE solution depends on a matrix we denote by $\hat{Q}$ and defined in [12]( $\mathbf{Q}$ depends on channel matrix and noise spectral density). Lets assume $\hat{\mathbf{Q}}$ has Cholesky factorization

$$
\hat{\mathbf{Q}}=L D L^{*}
$$

Then the feedback matrix $\mathbf{B}$ is found as a result of the above Cholesky factorization to be

$$
\mathbf{B}=L^{-1}
$$

The feedforward matrix $\mathbf{W}$ then is found from $\mathbf{B}$ (please refer to [12]). 


\subsubsection{Matrix Cholesky Factorization}

As discussed in the previous section the finite horizon (finite length) case, the MIMO DFE solution leads to a matrix Cholesky Factorization problem. We will use a similar state space approach used in the previous section to solve the matrix Cholesky factorization problem. Interestingly the statespace solution will lead to a Kalman filter solution to the matrix Cholesky factorization which in turn can be used to solve the Cholesky factorization encountered in the MIMO DFE problem.

Similar to the previous section we will present a Cholesky factorization solution for a vector process whose statistics is described by a state space model. Then in the next section we will formulate the DFE problem, and will use the development in this section to solve the corresponding DFE Cholesky factorization problem.

Lets assume a process $\left\{y_{i}\right\}$ with a time-varing spacetime model

$$
\left\{\begin{aligned}
x_{i+1} & =F_{i} x_{i}+G_{i} u_{i} \\
y_{i} & =H_{i} x_{i}+v_{i}
\end{aligned}\right.
$$

where $F_{i} \in C^{n \times n}, G_{i} \in C^{n \times m}$, and $H_{i} \in C^{p \times n}$ are known time-dependent matrices, and $\left\{u_{i}\right\}$ and $\left\{v_{i}\right\}$ are zeromean jointly stationary vector random variables that, along with the zero-mean random variable $x_{0}$ satisfy the conditions

$$
\left\langle\left[\begin{array}{c}
x_{0} \\
u_{i} \\
v_{i}
\end{array}\right],\left[\begin{array}{c}
x_{0} \\
u_{j} \\
v_{j} \\
1
\end{array}\right]\right\rangle=\left[\begin{array}{ccc}
\Pi_{0} & 0 & 0 \\
0 & {\left[\begin{array}{cc}
Q_{i} & S_{i} \\
S_{i} & R_{i}
\end{array}\right] d_{i, j}} & 0
\end{array}\right] .
$$

As seen the driving processes $\left\{u_{i}\right\}$ and $\left\{v_{i}\right\}$ are white (temporally uncorrelated). For more information on the above state-space description please refer to [14].

Lets assume we collect the process samples $\left\{y_{i}, i=\right.$ $1, \ldots, N\}$ in a vector $y$.

$$
y=\operatorname{col}\left\{y_{0}, y_{1}, \ldots, y_{N}\right\}
$$

and lets assume $R_{y}=\langle y, y\rangle$. The Cholesky factorization of $R_{y}$ seeks a factorization of the form

$$
R_{y}=L D L^{*}
$$

where $D$ is diagonal, and $L$ is lower triangular. If we also impose the condition that $L$ is lower triangular with unit diagonal, then under the additional assumption that all leading minors of $R_{y}$ are non-zero, it turns out that such factorization always exists and are also unique. A white noise process can be obtained from the factorization

$$
e=L^{-1} y, R_{e}=L^{-1} R_{y} L^{-*}=D
$$

where the vector $e=\operatorname{col}\left\{e_{0}, e_{1}, \ldots, e_{N}\right\}$ is also called the innovations process.

Now assuming that the $\left\{y_{i}\right\}$ process is modeled in statespace form (14), then the innovations process and therefore the Cholesky factorization of $R_{y}$ can be found through the Kalman filter recursions [14]. Here we will refer to a theorem summarizing the final results of the Kalman filter recursions as developed in [14].

Theorem 2 (The Kalman filter recursions for the innovations Consider the state-space equations

$$
\left\{\begin{aligned}
x_{i+1} & =F_{i} x_{i}+G_{i} u_{i} \\
y_{i} & =H_{i} x_{i}+v_{i}
\end{aligned}\right.
$$

where the $\left\{u_{i}, v_{i}, x_{0}\right\}$ are $m \times 1, p \times 1$, and $n \times 1$-dimensional random variables such that

$$
\left\langle\left[\begin{array}{c}
x_{0} \\
u_{i} \\
v_{i}
\end{array}\right],\left[\begin{array}{c}
x_{0} \\
u_{j} \\
v_{j} \\
1
\end{array}\right]\right\rangle=\left[\begin{array}{ccc}
\prod_{0} & 0 & 0 \\
0 & {\left[\begin{array}{cc}
Q_{i} & S_{i} \\
S_{i} & R_{i}
\end{array}\right] d_{i, j}} & 0
\end{array}\right]
$$

The matrices $\left\{F_{i}, G_{i}, H_{i}, \Pi_{0}, Q_{i}, S_{i}, R_{i}\right\}$ are assumed known. Then the lower triang ular canonical factor $L$ in the Cholesky factorization of $R_{y}$ is given by:

$$
L(i, j)=\left\{\begin{array}{cc}
0 & i<j \\
H_{i} \Phi(i-1, j-1) K_{p, j-1}, & i>j \\
I, & i=j
\end{array}\right.
$$

where

$$
\begin{gathered}
\Phi(i, j)=\left\{\begin{array}{cc}
F_{i+1} \cdots F_{j}, & i>j \\
I, & i=j
\end{array}\right. \\
K_{p, i}=\left(F_{i} P_{i} H_{i}^{*}+G_{i} S_{i}\right) R_{e, i}^{-1} \\
R_{e, i}=R_{i}+H_{i} P_{i} H_{i}^{*}
\end{gathered}
$$

$P_{i}=F_{i} P_{i} F_{i}^{*}+G_{i} Q_{i} G_{i}^{*}-K_{p, i} R_{e, i} K_{p, i}^{*} \quad i>0, P_{0}=\Pi_{0}$

We note that, $P_{i}=\left\langle\tilde{x}_{i}, \tilde{x}_{i}\right\rangle$, where $\tilde{x}_{i}=x_{i}-\bar{x}_{i}$

\subsubsection{Finite Horizon DFE solution}

As discussed in the section 2.2 the solution for the feedback section is found from Cholesky factorization of $\hat{\mathbf{Q}}$.

In the previous section (2.2.1), we showed how to factorize $R_{y}$ the covariance matrix of the stationary process $y$ which is described by a state-space model (14).

In order to use the solution obtained in the previous section we will equate

$$
R_{y} \triangleq \hat{\mathbf{Q}}
$$


We have the solution to factorizing $R_{y}$. Now we have to find the state-space model that leads to (20).

We will present the results and will leave the proof to [15]. It can be shown [15] that the following choices for the state-space model (14) will lead to (20):

$$
\begin{aligned}
& F_{i}=\left[\begin{array}{ccccc}
0 & 0 & \cdots & & 0 \\
I_{m} & 0 & \cdots & & \vdots \\
0 & I_{m} & 0 & & \\
\vdots & & \ddots & \ddots & \vdots \\
0 & \cdots & 0 & I_{m} & 0
\end{array}\right] \\
& H_{i}=\left[\begin{array}{llll}
\hat{Q}_{2} & \hat{Q}_{3} & \cdots & \hat{Q}_{l}
\end{array}\right] \\
& G_{i}=\left[\begin{array}{lll}
I_{m} & 0 \cdots
\end{array}\right]^{\prime} \\
& Q_{i}=0, \quad R_{i}=\hat{Q}_{11}+\frac{I}{S N R}, \quad S_{i}=I \text {. }
\end{aligned}
$$

where $l$ is the length of $\hat{\mathbf{Q}}$ in time. Therefore the DFE feedback matrix is $\mathbf{B}=L^{-1}$ where $L$ is found from Theorem 2 using the above state-space model parameters. The feedforward matrix $\mathbf{W}$ is then found from $\mathbf{B}$ (please refer to [12]). The key observation used in the proof is that the matrix $\mathbf{Q}$ has a block banded structure:

$\hat{\mathbf{Q}}=\left[\begin{array}{cccccccccc}\hat{Q}_{1} & \hat{Q}_{2} & \ldots & \hat{Q}_{l} & 0 & \ldots & \ldots & \ldots & \ldots & 0 \\ \hat{Q}_{2} & \hat{Q}_{1} & \hat{Q}_{2} & \ldots & \hat{Q}_{l} & 0 & & & & \\ \hat{Q}_{l} & \ldots & \hat{Q}_{2} & \hat{Q}_{1} & \hat{Q}_{2} & \ldots & \hat{Q}_{l} & 0 & & \\ 0 & \hat{Q}_{l} & \ldots & \hat{Q}_{2} & \hat{Q}_{1} & \hat{Q}_{2} & \ldots & \hat{Q}_{l} & 0 & \\ 0 & 0 & & & & & & & & \\ & & & & & & \hat{Q}_{l} & \ldots & \hat{Q}_{2} & \hat{Q}_{1}\end{array}\right]$

\section{CONCLUSION}

In this paper we proposed and investigated a new estimationbased approach to implementing a spatio-temporal Decision Feedback Equalizer (DFE) for MIMO (Multiple-Input Multiple-Output) channels. Both finite-length (Finite Horizon) and infinite-length (Infinite Horizon) MIMO Decision Feedback Equalizers were considered. For the infinite-length case the DFE problem lead to solving a matrix spectral factorization. For the finite-length case the DFE problem lead to solving a corresponding Cholesky factorization. Using the estimation-based spectral factorization we showed that the solution to the infinite-length MIMO DFE is not unique. In the finite-length case the estimation-based approach lead to a recursive algorithm to perform the Cholesky factorization. The proposed recursive algorithm is low complexity and is also simple to implement. Moreover it leads to a closed form solution for the MIMO DFE matrices.

\section{REFERENCES}

[1] G.J. Foschini. Layered Space-time Architecture for Wireless Communication in a fading environments using multi'element antennas. Bell Labs Technical Journal, 1(2):41-59, Autumn 1996.

[2] G.G. Raleigh and J.M. Cioffi. Spatio-temporal Coding for Wireless Communications. IEEE GLOBECOM 96, 3:180914, 1996.

[3] V. Tarokh and N. Seshadri and A. R. Calderbank. Spacetime codes for high data rate wireless communication: performance criterion and code construction. IEEE TRANSACTIONS ON INFORMATION THEORY, 44:744-65, 1998.

[4] B.C. Ng, J. Chen, and A. Paulraj. Space Time Processing for Fast Fading channels with Co-channel Interference. IEEE 46th Vehicular Technology Conference, 3:1491-5, 1996.

[5] S. Roy, J. Yang, and P.S. Kumar. Joint Transmitter/Receiver Optimization for Multiuser Communication. In W. A. Gardner, editor, Cyclostationarity in Communications and Signal Processing. IEEE Press, 1994.

[6] G.W. Wornell. Signal Processing Techniques for Efficien$\mathrm{t}$ Use of Transmit Diversity in Wireless Communications. 1996 IEEE International Conference on Acoustics, Speech, and Signal Processing, 2:1057-60, 1996.

[7] A. Duel-Hallen. Equalizers for Multiple Input Multiple Output Channels and PAM Systems with Cyclostationary Input Sequences. IEEE Journal on Selected Areas in Communications, 10:630-39, April 1992.

[8] J. Saltz. Digital Transmission Over Cross-Coupled Linear Channels. AT \&T Technical Journal, 64:1147-1159, JulyAugust 1985.

[9] Y. Kim and S. Shamsunder. Multichannel algorithms for Simultaneous Equalization and Interference Suppression. Wireless Personal Communications, 8:219-37, 1998.

[10] Ye $\mathrm{Li}$ and K. J. ray Liu. Adaptive Blind source Separation and Equalization for Multiple-Input/Multiple-Output Systems. IEEE Transactions on Information Theory, 44:28642876, 1998

[11] L. Vandendorpe and O. van de Wiel. MIMO DFE Equalization for Multitone DS/SS Systems over Multipath Channels. IEEE Journal on Selected Areas in Communications, 14:502-511, 1996.

[12] A. Maleki Tehrani, B. Hassibi, and J. Cịoffi. A New Estimation-based Approach to Spatio-Temporal MIMO Decision Feedback Equalization. Submitted to IEEE Vehicular Technology Conference 2000, 2000.

[13] B. Hassibi, A.H. Sayed, and T. Kailath. Indefinite Quadratic Estimation and Control. SIAM Studies in Applied Mathematics, 1998.

[14] T. Kailath, B. Hassibi, and A.H. Sayed. State Space Estimation. Printice Hall, 1999.

[15] A. Maleki Tehrani, B. Hassibi, and J. Cioffi. A New Estimation-based Approach to Spatio-Temporal MIMO Decision Feedback Equalization. Journal Paper, under preparation. 